\title{
Avaliação da presença e extensão dos atributos da atenção primária em Curitiba
}

\author{
Evaluation of the presence and extension of the attributes of primary care in Curitiba \\ Evaluación de la presencia y extensión de los atributos de la atención primaria en Curitiba
}

\author{
Eliane Chomatas. Secretaria Municipal da Saúde de Curitiba. Curitiba, PR, Brasil. elianechomatas@terra.com.br (Autora correspondente) \\ Alvaro Vigo. Universidade Federal do Rio Grande do Sul (UFRGS). Porto Alegre, RS, Brasil. alvaro.vigo@gamil.com \\ Inês Marty. Secretaria Municipal da Saúde de Curitiba. Curitiba, PR, Brasil. ineskm@hotmail.com \\ Lisiane Hauser. Universidade Federal do Rio Grande do Sul (UFRGS). Porto Alegre, RS, Brasil. lisiane.hauser@ymail.com \\ Erno Harzheim. Universidade Federal do Rio Grande do Sul (UFRGS). Porto Alegre, RS, Brasil. ernoharz@terra.com.br
}

\section{Resumo}

Os atributos da atenção primária à saúde (APS) - acesso, Iongitudinalidade, integralidade, coordenação, orientação familiar e comunitária e competência cultural - são reconhecidos como eixos estruturantes do processo de atenção, associados à qualidade dos serviços, à efetividade e à eficiência de suas intervenções. Foram investigadas a presença e a extensão dos atributos da APS no município de Curitiba, comparando unidades do modelo tradicional e Estratégia Saúde da Família (ESF), mediante 0 instrumento PCATool-Brasil aplicado a 490 médicos e enfermeiros. Os resultados indicam que na ESF os escores médios e o percentual de Alto Escore $(\geq 6,6)$ dos atributos longitudinalidade, integralidade dos serviços disponíveis e prestados, orientação familiar e orientação comunitária são significativamente maiores que nas unidades tradicionais, assim como para os atributos condensados nos escores Essencial, Derivado e Geral da APS. Os resultados evidenciam que em Curitiba as unidades com ESF possuem maior presença e extensão dos atributos da APS.

\section{Abstract}

The attributes of Primary Health Care (PHC): access to first contact, longitudinally, comprehensiveness, coordination, family and community orientation, and cultural competence are recognized as the structural pillars of health care process, associated with service quality and intervention effectiveness and efficiency. We compared health centers that operate with the traditional model with those with Family Health Strategy (FHS), by means of the instrument PCATool-Brazil, applied to 490 doctors and nurses. In FHS, the average scores and the percentage of high scores ( $\geq 6.6)$ in relation to the attributes longitudinally, comprehensiveness of available services and services provided, family orientation, and community orientation are significantly higher than in traditional health centers. The same occurs when these attributes are condensed as PHC Essential, Derived or General Scores. Results demonstrate that the FHS health centers in Curitiba have greater presence and extension of primary care attributes.

\section{Resumen}

Los atributos de atención primaria de salud (APS) - acceso, longitudinalidad, integralidad, coordinación, orientación familiar y comunitaria y competencia cultural - son reconocidos como ejes estructurantes del proceso de atención, asociados a la calidad de los servicios, a la efectividad y a la eficiencia de sus intervenciones. Se investigó la presencia y la extensión de los atributos de la APS en el municipio de Curitiba, comparando unidades del modelo tradicional con unidades de la Estrategia de Salud Familiar (ESF), mediante el instrumento PCATool-Brasil aplicado a 490 médicos y enfermeros de la APS. Los resultados indican que en la ESF los puntajes medios y el porcentaje de alta puntuación $(\geq 6,6)$ de los atributos longitudinalidad, integralidad de los servicios disponibles y de los servicios prestados, orientación familiar y orientación comunitaria son significativamente mayores que en las unidades tradicionales, así como los atributos agrupados en los puntajes Esencial, Derivado y General de la APS. Los resultados evidencian que en Curitiba las unidades con ESF tienen una mayor presencia y extensión de los atributos de la APS.

Palavras-chave: Atenção Primária à Saúde Avaliação de Serviços de Saúde Programa Saúde da Família

\section{Keywords:}

Primary Health Care Health Services Evaluation Family Health Program

\section{Palabras clave:}

Atención Primaria de Salud Evaluación de Servicios de Salud Programa de Salud Familiar

Fonte de financiamento: Secretaria Municipal da Saúde de Curitiba - PR.

Parecer CEP: Secretaria Municipal de Saúde de Curitiba, n. 21/2008.

Conflito de interesses: declaram não haver. Recebido em: 03/05/2013 Aprovado em: 08/10/2013 


\section{Introdução}

A Atenção Primária à Saúde (APS) é conceituada como o primeiro nível de assistência dentro do sistema de saúde, caracterizando-se principalmente pela longitudinalidade e a integralidade da atenção, além da coordenação da assistência dentro do próprio sistema, da atenção centrada na família, da orientação e participação comunitária e da competência cultural. Essa operacionalização tem sido utilizada para definir os quatro atributos essenciais da APS (o acesso do primeiro contato do indivíduo com o sistema de saúde, a longitudinalidade, a integralidade e a coordenação da atenção), bem como os três atributos derivados que qualificam suas açôes (a orientação centrada na família, a orientação comunitária e a competência cultural $)^{1-3}$.

Na década de 1970 e início dos anos 1980, ecoavam no Brasil os princípios de Alma Ata, que encontraram no Movimento Brasileiro pela Reforma Sanitária um forte aliado ${ }^{4}$. Esse movimento, lutando pela implantação de um sistema gratuito e universal, teve sua expressão máxima na $8^{a}$ Conferência Nacional de Saúde ${ }^{5}$, quando foram delineados os princípios norteadores do Sistema Único de Saúde (SUS), mais tarde incorporados na Constituição Federal de $1988^{6}$. A partir desse momento, a APS foi se consolidando como o ponto de atenção preferencial do primeiro contato do indivíduo com os serviços de saúde. Em 1991, foi implantado pelo governo federal o Programa de Agentes Comunitários de Saúde 7 e, em 1994, o Programa Saúde da Família, posteriormente denominado Estratégia Saúde da Família ${ }^{8}$.

Regida pelos princípios da Conferência de Alma Ata e da APS, Curitiba iniciou a implantação de uma rede de unidades de saúde voltada ao acesso da populaçáo de baixa renda, com definiçáo de área de abrangência, buscando garantia da qualidade, eficiência do atendimento e priorizando programas (gestantes, crianças, adultos, hipertensos) ${ }^{9}$. Em 1993, com a inauguração da Unidade de Saúde São José, o município de Curitiba implantou sua primeira unidade de Saúde da Família ${ }^{10}$. Em 1996, Curitiba aderiu à ESF recebendo parte do custeio das equipes do governo federal, o que permitiu ao município a expansão desse modelo de atenção. Em 2008, existiam 53 unidades básicas com ESF e 51 unidades básicas tradicionais (UTRAD).

Em 2008, ambos os modelos atuavam na filosofia da APS, porém as unidades com ESF possuíam médicos generalistas, alguns com especialidade em Medicina de Família e Comunidade (MFC), que, assim como os demais profissionais da equipe, trabalhavam oito horas diárias, desenvolvendo atividades internas e externas da unidade de saúde (US). As UTRAD atuavam com médicos das especialidades básicas (pediatras, gineco-obstetras e clínicos) que trabalhavam quatro horas diárias nas atividades internas das US. Nos dois modelos, existiam consultas de demanda espontânea e consultas vinculadas aos programas estratégicos da Secretaria Municipal de Saúde (SMS), assim como agentes comunitários de saúde, áreas de abrangência subdivididas em microáreas, e prática do conceito de vigilância em saúde ${ }^{11}$.

Partindo dos referenciais teóricos apresentados e utilizando metodologia universalmente aceita, este estudo tem como objetivo avaliar a presença e a extensão dos atributos da atenção primária à saúde no município de Curitiba, comparando unidades tradicionais com unidades da Estratégia Saúde da Família, por meio da experiência de seus profissionais de saúde.

\section{Métodos}

\section{Delineamento}

Foi realizado um estudo transversal utilizando o Instrumento de Avalição da APS - PCATool-Brasil, aplicado aos servidores médicos e enfermeiros das unidades de saúde. Os dados foram coletados no período de maio a novembro de 2008, no município de Curitiba, Paraná, Brasil.

\section{População e amostra}

À época do estudo, existiam no município 104 unidades básicas de saúde, sendo 53 com ESF e 51 tradicionais. As unidades com ESF estavam distribuídas nos nove distritos sanitários do município, mas de forma heterogênea. Por exemplo, no Distrito Sanitário do Bairro Novo, todas as US atuavam com ESF, enquanto no Distrito Sanitário do Boa Vista, que possuía 15 unidades básicas, apenas 3 atuavam com ESF. As unidades com ESF foram implantadas em áreas de maior vulnerabilidade socioeconômica, com o propósito de reverter os indicadores epidemiológicos. 
Foram selecionadas as unidades implantadas antes de junho de 2006 e que atuavam exclusivamente com atenção primária. Assim, participaram do estudo 45 unidades que atuavam com ESF (84,9\% do total das unidades com ESF) e 47 UTRAD ( $92,1 \%$ do total das unidades tradicionais).

O instrumento PCATool-Brasil foi aplicado aos profissionais médicos pediatras, gineco-obstetras, clínicos gerais e enfermeiros das unidades tradicionais, bem como aos profissionais médicos que atuavam como generalistas, médicos de família e comunidade e enfermeiros das unidades que trabalhavam com a ESF.

\section{Descrição do PCATool}

Na Johns Hopkins University, entre 1997 e 2001, Barbara Starfield e colaboradores desenvolveram o Primary Care Assessment Tool (PCATool), um conjunto de instrumentos de avaliação da atenção primária em duas versóes destinadas aos usuários crianças $^{1}$ e adultos ${ }^{12}$ e uma terceira destinada aos profissionais de saúde ${ }^{1}$. Esse instrumento é baseado em diversos itens e tem como objetivo medir a presença e a extensão dos seguintes atributos: acesso ao primeiro contato, longitudinalidade, coordenação, integralidade, orientação familiar e orientação comunitária. As duas versôes do PCATool (saúde da criança e do adulto) para aplicação aos usuários dos serviços de saúde já foram traduzidas e validadas no Brasil ${ }^{13,14}$. O instrumento destinado aos profissionais de saúde utilizado neste estudo é a versão em espelho da versão validada para usuários adultos. O questionário foi elaborado para coletar informaçôes a respeito das características operacionais e práticas - de estrutura e do processo de atenção - relacionadas à atenção primária desenvolvida por profissionais envolvidos na assistência direta aos usuários.

A aplicação do instrumento permite a construção de escores para cada dimensão (atributo) e seus componentes. As respostas sáo estruturadas seguindo uma escala do tipo Likert, atribuindo escores no intervalo de 1 a 4 para cada atributo ( 1 = com certeza não; 2 = provavelmente não; 3 = provavelmente sim; e 4 = com certeza sim). Para obter o escore da qualidade da APS, foi calculada a média dos valores dos itens que compóem cada atributo e seus componentes.

O Escore Essencial foi obtido por meio da média dos atributos essenciais (acesso, longitudinalidade, coordenação e integralidade), o escore derivado foi obtido pela média dos atributos derivados (orientação familiar e orientação comunitária) e o Escore Geral da APS, pelo valor médio dos atributos essenciais e dos atributos derivados. O valor obtido para estes escores foram então transformados em uma escala entre 0 e 10 , como se segue ${ }^{15}$ :

$$
\text { Escore geral da APS }=\frac{(\text { Escore obtido }-1) \times 10}{4-1}
$$

Os escores dos atributos, Geral, Essencial e Derivado foram, entấo, dicotomizados em duas categorias, representando Baixo Escore, quando $<6,6$, e Alto Escore, quando $\geq 6,6$.

\section{Aplicação do PCATool}

Os questionários foram aplicados por alunos do curso de medicina da Pontifícia Universidade Católica do Paraná (PUC-PR). Todos os entrevistadores foram treinados, sendo realizado um piloto do estudo nas unidades básicas excluídas da amostra.

No decorrer da pesquisa, foram excluídas outras duas US - uma por apresentar profissionais vinculados a outra instituição (PUC-PR) e outra por apresentar dados secundários, utilizados no segundo tópico da pesquisa (apresentado em outro artigo), não confiáveis. Um questionário foi excluído por apresentar ausência de respostas para a maioria das perguntas.

\section{Análise estatística}

Os questionários foram digitados em Epidata, versão 3.1. Todas as análises foram realizadas com o programa SAS, versão 9.1.3. As comparaçôes de médias foram realizadas mediante o teste t para amostras independentes com estimativa conjunta das variâncias ou utilizando o método de Satterthwaite para variâncias populacionais diferentes. As comparaçôes entre proporções foram realizadas utilizando o teste baseado na estatística $\chi^{2}$ de Pearson com correção de Yates, considerando 
amostras independentes. A associação entre a presença de Alto Escore de APS com os preditores foi estimada usando o modelo de regressão de Poisson robusta ${ }^{16}$.

\section{Aspectos éticos}

O estudo foi submetido e aprovado pelo Comitê de Ética em Pesquisa da Secretaria Municipal de Saúde de Curitiba, sob número 21/2008, seguindo os preceitos éticos. Todos os entrevistados concordaram em participar da pesquisa e leram, aprovaram e assinaram o Termo de Consentimento Livre e Esclarecido (TCLE).

\section{Resultados}

Entre os 263 profissionais de ESF elegíveis, 190 (72\%) responderam, sendo 91 médicos e 99 enfermeiros, representando respectivamente $48 \%$ e $52 \%$ da amostra da ESF. Dos 442 profissionais de UTRAD elegíveis, $300(67,8 \%)$ responderam os questionários, 223 dos respondentes sendo médicos (74\%) e 77 (26\%), enfermeiros.

A Tabela 1 descreve as características da amostra. A idade média dos médicos das UTRAD foi de 42,5 (IC95\%: 41,2-43,8) e nas unidades com ESF foi de 42,6 (IC95\%: 40,0-45,1). Para os enfermeiros, as idades médias nas UTRAD e nas unidades com ESF foram de 35,7 anos (IC95\%: 34,0-37,3) e 39,2 anos (IC95\%: 37,5-40,9), respectivamente. Considerando-se a amostra global, $420(85,7 \%)$ profissionais declararam ter cor da pele branca. O tempo médio de formados dos médicos foi de 17,8 anos (IC95\%: 16,4-19,1) nas UTRAD e 17,8 (IC95\%: 15,6-20,1) nas unidades com ESF, enquanto que para os enfermeiros foi respectivamente 9,4 anos (IC95\%: 7,8-11,1) e 13,0 anos (IC95\%: 11,3-14,6). Ainda em relação à formação nas UTRAD, 199 (89,2\%) médicos e 46 (59,7\%) enfermeiros referiram ter alguma especialização e nas ESF os números encontrados foram $70(76,9 \%)$ e $63(63,6 \%)$, respectivamente.

Os médicos das unidades com ESF dedicaram à educação comunitária, em média, 5,3\% (IC95\%: 4,0-6,6) da carga horária, mais que o dobro da carga dos médicos das UTRAD, igual a 2,5\% (IC95\%: 1,5-3,4) ( $\mathrm{p}=0,01)$. Para as visitas domiciliares, esses percentuais foram ainda mais discrepantes, sendo 8,8\% (IC95\%: 7,5-10,1) e 1,2\% (IC95\%: 0,5-1,9) respectivamente para unidades com ESF e as UTRAD $(\mathrm{p}<0,01)$. Similarmente, o percentual da carga horária despendido para discussão de casos com a equipe foi de 1,9\% (IC95\%: 1,2-2,7) para os médicos das UTRAD, contra 5,1\% (IC95\%; 3,8-6,5) nas unidades com ESF $(\mathrm{p}<0,01)$.

Em relação ao perfil da pós-graduação realizada pelos profissionais que atuavam na APS, 10 médicos (11\%) que atuavam nas unidades com ESF fizeram residência em medicina de família e comunidade, 27(29,7\%) possuíam título em medicina de família e comunidade e $19(20,9 \%)$ possuíam especialização em saúde da família, enquanto nas UTRAD foram observados 7 (3,1\%), $3(1,3 \%)$ e 8 (3,6\%), respectivamente. Similarmente, entre os enfermeiros das equipes com ESF, 18 (18,2\%) tinham especialização em saúde da família, e $3(3,0 \%)$ realizaram especialização ou residência em enfermagem comunitária, enquanto nas UTRAD 10 (13\%) possuíam especialização em saúde da família.

Para todos os quesitos avaliados, os profissionais afirmaram ter alto grau de satisfação em relação ao trabalho, ao coordenador, à equipe e à comunidade (Tabela 2). O menor grau de satisfação $(73,6 \%)$ foi observado entre os médicos das unidades que atuavam com ESF em relação ao coordenador, enquanto o maior ocorreu no quesito satisfação com a comunidade $(92,9 \%)$ nas respostas dos enfermeiros que atuavam nas ESF.

A Tabela 3 resume as informações relativas à avaliação dos profissionais sobre atividades de capacitação, presença de violência na comunidade e grau de motivação e criatividade em relação ao serviço. Nas unidades com ESF, 91,2\% dos profissionais médicos e $92,9 \%$ dos enfermeiros afirmaram que o serviço ofertava capacitação, enquanto nas unidades tradicionais os valores encontrados foram 69,5\% e 90,9\%, respectivamente. Ainda, 95,6\% dos médicos e 89,9\% dos enfermeiros das unidades com ESF indicaram ter realizado capacitação na sua área de atuação no último ano, percentuais similares aos observados nas unidades tradicionais ( $88,8 \%$ dos médicos e $88,3 \%$ dos enfermeiros).

Os médicos e enfermeiros das unidades com ESF e os enfermeiros das UTRAD indicaram a capacitação em serviço como a principal estratégia de capacitação, enquanto entre os médicos das UTRAD a participação em congressos foi mais citada. Em relação à percepção do grau de violência na comunidade em que atuavam, 26,9\% dos médicos e 40,3\% enfermeiros 
das unidades tradicionais relataram que era alto, enquanto nas unidades com ESF esses valores foram 60,4\% e 73,7\%, respectivamente. Altos índices de motivação, criatividade e orgulho pelo trabalho realizado foram apontados pela maioria dos profissionais nos dois modelos de atenção.

Tabela 1. Média (EP†) ou n (\%) das características dos profissionais médicos e enfermeiros da rede de atenção primária à saúde de Curitiba de acordo com o tipo de unidade de saúde, 2008.

\begin{tabular}{|c|c|c|c|c|}
\hline \multirow{3}{*}{ Característica } & \multicolumn{2}{|c|}{ Médicos } & \multicolumn{2}{|c|}{ Enfermeiros } \\
\hline & UTRAD & ESF & UTRAD & ESF \\
\hline & $(\mathrm{N}=223)$ & $(\mathrm{N}=91)$ & $(\mathrm{N}=77)$ & $(\mathrm{N}=99)$ \\
\hline \multicolumn{5}{|l|}{ Sexo } \\
\hline Masculino & $89(39,9)$ & $51(56,0)$ & $4(5,2)$ & $7(7,1)$ \\
\hline Feminino & $134(60,1)$ & $40(44,0)$ & $73(94,8)$ & $92(92,9)$ \\
\hline \multicolumn{5}{|l|}{ Cor da pele } \\
\hline Branca & $193(86,5)$ & $78(85,7)$ & $66(85,7)$ & $83(83,8)$ \\
\hline Não branca & $30(13,5)$ & $13(14,3)$ & $11(14,3)$ & $16(11,1)$ \\
\hline \multicolumn{5}{|l|}{ Estado civil } \\
\hline Casado & $146(65,5)$ & $58(63,7)$ & $50(64,9)$ & $64(64,6)$ \\
\hline Outros & $77(34,5)$ & $33(36,3)$ & $27(35,1)$ & $35(35,40)$ \\
\hline \multicolumn{5}{|l|}{ Especialização } \\
\hline Sim & $199(89,2)$ & $70(76,9)$ & $46(59,7)$ & $63(63,6)$ \\
\hline Não & $24(10,8)$ & $24(23,1)$ & $31(40,3)$ & $36(36,4)$ \\
\hline Residência em medicina de família e comunidade & $7(3,1)$ & $10(11,0)$ & - & - \\
\hline Prova de título em medicina de família e comunidade & $3(1,4)$ & $27(29,7)$ & - & - \\
\hline Residência ou prova de título em clínica médica & $16(7,2)$ & $7(7,7)$ & - & - \\
\hline Residência ou prova de título em pediatria & $87(39,0)$ & $12(13,2)$ & - & - \\
\hline Especialização em saúde pública & $6(2,7)$ & $8(8,8)$ & $6(7,8)$ & $12(12,1)$ \\
\hline Especialização em saúde da família & $8(3,6)$ & $19(20,9)$ & $10(13,0)$ & $18(18,2)$ \\
\hline Residência ou prova de título em ginecologia-obstetrícia & $51(22,9)$ & $7(7,7)$ & - & - \\
\hline Especialização ou residência em enfermagem comunitária & - & - & - & $3(3,0)$ \\
\hline Residência em medicina social & - & $1(1,1)$ & - & - \\
\hline Residência multiprofissional & $2(0,9)$ & - & - & - \\
\hline Outras especializações* & $18(8)$ & $6(6,6)$ & $9(11,7)$ & $7(7,6)$ \\
\hline Idade (anos) & $42,5(0,65)$ & $42,6(1,28)$ & $35,7(0,83)$ & $39,2(0,86)$ \\
\hline Tempo de formado (anos) & $17,8(0,70)$ & $17,8(1,13)$ & $9,4(0,82)$ & $13,0(0,84)$ \\
\hline Número de locais de trabalho & $2,9(0,07)$ & $1,8(0,10)$ & $1,3(0,06)$ & $1,1(0,03)$ \\
\hline Número de horas de trabalho/semana & $50,1(1,15)$ & $50,2(1,29)$ & $46,2(1,45)$ & $42,7(0,97)$ \\
\hline \multicolumn{5}{|l|}{ Tempo de serviço (anos) } \\
\hline US & $5,8(0,42)$ & $5,2(0,55)$ & $2,0(0,29)$ & $4,0(0,49)$ \\
\hline SMS & $8,1(0,51)$ & $10,1(0,81)$ & $3,5(0,56)$ & $6,8(0,58)$ \\
\hline Número médio de consultas na semana & $74,3(1,45)$ & $121,2(2,35)$ & $44,3(3,03)$ & $50,0(2,78)$ \\
\hline \multicolumn{5}{|l|}{ \% de carga horária semanal } \\
\hline 20 horas & $217(97,3)$ & - & $2(2,6)$ & - \\
\hline 40 horas & $6(2,6)$ & $91(100)$ & $75(97,4)$ & $99(100)$ \\
\hline \multicolumn{5}{|l|}{ \% de carga horária semanal por atividade } \\
\hline Educação comunitária & $2,5(0,48)$ & $5,3(0,64)$ & $10,4(1,33)$ & $11,3(0,94)$ \\
\hline Visitas domiciliares & $1,2(0,36)$ & $8,8(0,65)$ & $8,2(1,14)$ & $11,9(0,70)$ \\
\hline Reuniões & $2,6(0,41)$ & $6,0(0,44)$ & $9,2(1,01)$ & $7,9(0,48)$ \\
\hline Consulta demanda & $44,6(1,79)$ & $41,4(2,01)$ & $31,0(2,48)$ & $27,8(1,54)$ \\
\hline Consulta programada & $47,3(1,82)$ & $33,4(1,78)$ & $34,3(2,67)$ & $33,2(1,74)$ \\
\hline Discussão de caso & $1,9(0,39)$ & $5,1(0,71)$ & $6,9(0,93)$ & $7,8(0,72)$ \\
\hline
\end{tabular}

†EP - Erro padrão da média. * - Especializações não direcionadas à APS (cardiologia, hematologia, neurologia etc.). 
Tabela 2. Frequência (\%) de satisfação dos profissionais de acordo com tipo de unidade de saúde e categoria profissional da rede de atenção primária à saúde de Curitiba, 2008.

\begin{tabular}{|c|c|c|c|c|c|c|}
\hline \multirow{2}{*}{ Satisfação } & \multicolumn{3}{|c|}{ Médicos } & \multicolumn{3}{|c|}{ Enfermeiros } \\
\hline & UTRAD & ESF & $p$-valors & UTRAD & ESF & $\mathrm{p}$-valors \\
\hline \multicolumn{7}{|l|}{ Com o trabalho } \\
\hline Satisfeito $^{\dagger}$ & $171(76,7)$ & $64(79,3)$ & & $64(83,1)$ & $79(79,8)$ & \\
\hline Não satisfeito* & $52(23,3)$ & $27(29,7)$ & 0,3014 & $13(16,9)$ & $20(21,2)$ & 0,7151 \\
\hline \multicolumn{7}{|l|}{ Com o coordenador } \\
\hline Satisfeito $^{\dagger}$ & $187(83,9)$ & $67(73,6)$ & & $68(88,3)$ & $80(80,8)$ & \\
\hline Não satisfeito* & $36(16,1)$ & $24(26,4)$ & 0,0532 & $9(11,7)$ & $19(19,2)$ & 0,2533 \\
\hline \multicolumn{7}{|l|}{ Com a equipe } \\
\hline Satisfeito $^{+}$ & $199(89,2)$ & $75(82,4)$ & & $68(88,3)$ & $89(89,9)$ & \\
\hline Não satisfeito* & $24(10,8)$ & $16(17,6)$ & 0,1449 & $9(11,7)$ & $10(10,1)$ & 0,9269 \\
\hline \multicolumn{7}{|l|}{ Com a comunidade } \\
\hline Satisfeito $^{\dagger}$ & $185(83,0)$ & $77(84,6)$ & & $61(79,2)$ & $92(92,9)$ & \\
\hline Não satisfeito* & $38(17,0)$ & $14(15,4)$ & 0,8487 & $16(2,8)$ & $7(7,1)$ & 0,0142 \\
\hline
\end{tabular}

${ }^{\dagger}$ Muito satisfeito ou satisfeito. *Indiferente ou insatisfeito ou muito insatisfeito. ${ }^{\$}$ Associado ao teste $\chi^{2}$ com correção de Yates.

Tabela 3. Frequência (\%) de avaliação dos profissionais médicos e enfermeiros da rede de atenção primária à saúde de Curitiba quanto a atividades de capacitação, percepção de violência na comunidade e grau de motivação e criatividade referentes ao serviço, 2008.

\begin{tabular}{|c|c|c|c|c|}
\hline \multirow{3}{*}{ Característica } & \multicolumn{2}{|c|}{ Médicos } & \multicolumn{2}{|c|}{ Enfermeiros } \\
\hline & UTRAD & ESF & UTRAD & ESF \\
\hline & $(\mathrm{N}=223)$ & $(\mathrm{N}=91)$ & $(\mathrm{N}=77)$ & $(\mathrm{N}=99)$ \\
\hline \multicolumn{5}{|l|}{ Serviço oferece formação continuada } \\
\hline Sim & $155(69,5)$ & $83(91,2)$ & $70(90,9)$ & $92(92,9)$ \\
\hline Não & $68(30,5)$ & $8(8,8)$ & $7(9,1)$ & $7(7,1)$ \\
\hline \multicolumn{5}{|c|}{ Realizou capacitação no último ano na área de atuação } \\
\hline Sim & $198(88,8)$ & $87(95,6)$ & $68(88,3)$ & $89(89,9)$ \\
\hline Não & $25(11,2)$ & $4(4,4)$ & $9(11,7)$ & $10(10,1)$ \\
\hline \multicolumn{5}{|l|}{ Tipo de capacitação realizada ${ }^{\dagger}$} \\
\hline Congresso & $146(65,5)$ & $58(63,7)$ & $13(16,9)$ & $16(16,2)$ \\
\hline Especialização & $57(25,6)$ & $24(26,4)$ & $8(10,4)$ & $22(22,2)$ \\
\hline Ensino a distância & $38(17,0)$ & $28(30,8)$ & $19(24,7)$ & $24(24,2)$ \\
\hline Capacitação em campo & $107(48,0)$ & $70(76,9)$ & $62(80,5)$ & $81(81,8)$ \\
\hline \multicolumn{5}{|l|}{ Percepção do grau de violência } \\
\hline Alto & $60(26,9)$ & $55(60,4)$ & $31(40,3)$ & $73(73,7)$ \\
\hline Médio/baixo & $163(73,1)$ & $36(39,6)$ & $46(59,7)$ & $26(26,3)$ \\
\hline \multicolumn{5}{|l|}{ Grau de motivação } \\
\hline Alta & $214(96,0)$ & $85(93,4)$ & $75(97,4)$ & $97(98,0)$ \\
\hline Baixa & $9(4,0)$ & $6(6,6)$ & $2(2,6)$ & $2(2,0)$ \\
\hline \multicolumn{5}{|l|}{ Grau de criatividade } \\
\hline Alta & $208(93,3)$ & $86(94,5)$ & $76(98,7)$ & $98(99,0)$ \\
\hline Baixa & $15(6,7)$ & $5(5,5)$ & $1(1,3)$ & $1(1,0)$ \\
\hline \multicolumn{5}{|l|}{ Sente-se orgulhoso do trabalho } \\
\hline Sim & $202(90,6)$ & $87(95,6)$ & $74(96,1)$ & $98(99,0)$ \\
\hline Não & $21(9,4)$ & $4(4,4)$ & $3(3,9)$ & $1(1,0)$ \\
\hline
\end{tabular}

${ }^{\dagger}$ Diferentes tipos de capacitação podem ter sido realizados no período pelo mesmo profissional.

A Tabela 4 mostra a presença e a extensão dos atributos da APS considerando os escores médios de cada atributo, o Escore Essencial, o Escore Derivado e o Escore Geral da APS separadamente para as unidades tradicionais ou com ESF. $\mathrm{Na}$ maioria dos atributos, os escores médios alcançados pelas unidades com ESF superaram os das UTRAD, embora nos atributos acessibilidade e coordenação a diferença não tenha sido estatisticamente significativa. As unidades com ESF também apresentaram maior percentual de alto escore $(\geq 6,6)$ da APS, tanto nos atributos individuais quanto nos Escores Essencial, Derivado e Geral da APS. 
Tabela 4. Escores ${ }^{\dagger}$ médios dos atributos e dos escores essencial, derivado e geral de atenção primária à saúde e frequência de alto escore $(\geq 6,6)$ na avaliação dos profissionais médicos e enfermeiros das unidades tradicionais e com Estratégia Saúde da Família do município de Curitiba, 2008.

\begin{tabular}{|c|c|c|c|c|c|c|}
\hline \multirow{3}{*}{ Atributo } & \multicolumn{3}{|c|}{ Escores médios (IC 95\%) } & \multicolumn{3}{|c|}{ Escore alto $(\geq 6,6)$} \\
\hline & UTRAD & ESF & P-valor ${ }^{\ddagger}$ & UTRAD & ESF & P-valor* \\
\hline & $(\mathrm{N}=300)$ & $(\mathrm{N}=190)$ & & $\mathrm{n}(\%)$ & $\mathrm{n}(\%)$ & \\
\hline \multicolumn{7}{|l|}{ Atributos da Atenção Primária } \\
\hline Acessibilidade & $4,1(3,9-4,2)$ & $4,2(4,0-4,4)$ & 0,4331 & $12(4,0)$ & $11(5,8)$ & 0,4933 \\
\hline Longitudinalidade & $6,0(5,8-6,1)$ & $6,6(6,4-6,7)$ & $<0,0001$ & $102(34,1)$ & $97(51,0)$ & 0,0003 \\
\hline Coordenação do Cuidado & $6,9(6,7-7,0)$ & $7,0(6,8-7,1)$ & 0,5301 & $191(63,7)$ & $127(66,8)$ & 0,5350 \\
\hline Coordenação Sistema Informação & $8,3(8,1-8,5)$ & $8,5(8,2-8,7)$ & 0,1625 & $264(88,0)$ & $171(90,5)$ & 0,4823 \\
\hline Integralidade/serviços disponíveis & $7,1(7,0-7,2)$ & $7,9(7,8-8,0)$ & $<0,0001$ & $217(72,3)$ & $171(90,0)$ & $<0,0001$ \\
\hline Integralidade/serviços prestados & $6,0(5,7-6,2)$ & $8,3(8,2-8,5)$ & $<0,0001$ & $150(50,0)$ & $172(90,5)$ & $<0,0001$ \\
\hline Essencial & $6,4(6,3-6,5)$ & $7,1(7,0-7,2)$ & $<0,0001$ & $122(40,7)$ & $142(74,7)$ & $<0,0001$ \\
\hline Orientação familiar & $7,8(7,6-8,0)$ & $8,5(8,3-8,7)$ & $<0,0001$ & $261(87,0)$ & $184(96,8)$ & 0,0004 \\
\hline Orientação comunitária & $7,6(7,4-7,8)$ & $8,1(7,9-8,3)$ & 0,0001 & $232(77,6)$ & $168(88,4)$ & 0,0037 \\
\hline Derivado & $7,7(7,5-7,9)$ & $8,3(8,2-8,5)$ & $<0,0001$ & $237(79,3)$ & $178(93,7)$ & $<0,0001$ \\
\hline Geral & $6,7(6,6-6,8)$ & $7,4(7,3-7,5)$ & $<0,0001$ & $167(55,7)$ & $163(85,8)$ & $<0,0001$ \\
\hline
\end{tabular}

${ }^{\dagger}$ Os escores assumem valores de $0-10 .{ }^{\ddagger}$ Obtido pelo teste $t$ para amostras independentes com estimativa conjunta das variâncias ou utilizando 0 método de Satterthwaite para variâncias diferentes. "Associado ao teste $\chi^{2}$ com correção de Yates.

Os resultados da regressão de Poisson robusta univariável e multivariável são mostrados na Tabela 5. Considerando que existe uma forte correlação entre idade e tempo de formação, apenas esta variável foi utilizada nos modelos multivariáveis. Quando as duas categorias profissionais foram avaliadas em conjunto (modelo geral), unidades com ESF (RP=1,44; IC95\%: 1,27-1,62) estavam associadas com um aumento da prevalência de Alto Escore Geral da APS, mesmo ajustando para tempo de formado, carga de trabalho semanal, tempo de serviço na SMS, ter realizado alguma especialização, profissão e formação em medicina de família ou enfermagem comunitária. Por outro lado, ser profissional médico em relação ao enfermeiro $(\mathrm{RP}=0,87$; IC95\%: 0,78-0,99) estava associado com uma diminuição da prevalência de Alto Escore Geral da APS.

Tabela 5. Associação ${ }^{\dagger}$ entre a presença de Alto Escore Geral da APS com seus preditores entre profissionais médicos e enfermeiros da rede de atenção primária à saúde de Curitiba, 2008.

\begin{tabular}{|c|c|c|c|c|c|c|}
\hline \multirow{3}{*}{ Preditores } & \multicolumn{2}{|c|}{$\begin{array}{c}\text { Modelo Geral: médicos e } \\
\text { enfermeiros }\end{array}$} & \multicolumn{2}{|c|}{ Modelo Estratificado: médicos } & \multicolumn{2}{|c|}{ Modelo Estratificado: enfermeiros } \\
\hline & Univariável & Multivariável & Univariável & Multivariável & Univariável & Multivariável \\
\hline & RP (IC 95\%) & RP (IC 95\%) & RP (IC 95\%) & RP (IC 95\%) & RP (IC 95\%) & RP (IC 95\%) \\
\hline Tipo de unidade (ESF x UTRAD) & $1,54(1,37-1,73)$ & $1,44(1,28-1,62)$ & $1,74(1,49-2,03)$ & $1,42(1,13-1,79)$ & $1,16(0,99-1,35)$ & $1,16(0,98-1,37)$ \\
\hline Sexo $(M \times F)$ & $0,87(0,75-1,00)$ & & $1,03(0,86-1,23)$ & & $0,78(0,49-1,22)$ & \\
\hline Idade (aumento de 10 anos) & $0,95(0,90-1,02)$ & & $0,99(0,91-1,08)$ & & $0,99(0,90-1,08)$ & \\
\hline 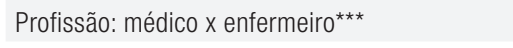 & $0,74(0,66-0,83)$ & $0,87(0,78-, 0,99)$ & & & & \\
\hline Especialização (sim x não) & $0,80(0,71-0,91)$ & $0,89(0,77-1,02)$ & $0,79(0,64-0,96)$ & & $0,94(0,82-1,09)$ & \\
\hline Tempo de formado (aumento de 5 anos) & $0,97(0,94-1,00)$ & $0,98(0,95-1,03)$ & $0,98(0,94-1,03)$ & $0,98(0,93-1,04)$ & $1,00(0,96-1,05)$ & $0,99(0,93-1,05)$ \\
\hline $\begin{array}{l}\text { Formação em medicina de família ou } \\
\text { enfermagem comunitária** }\end{array}$ & $1,19(1,04-1,36)$ & $1,08(0,93-1,25)$ & & & & \\
\hline Formação em medicina de família* & & & $1,43(1,21-1,69)$ & $1,06(0,88-1,28)$ & & \\
\hline Formação em enfermagem comunitária*** & & & & & $1,24(1,16-1,34)$ & $1,16(1,05-1,29)$ \\
\hline Carga de trabalho semanal (aumento de $1 \mathrm{~h}$ ) & $1,00(0,99-1,00)$ & $1,00(0,99-1,00)$ & $1,00(0,99-1,00)$ & $1,00(0,99-1,00)$ & $1,00(0,99-1,01)$ & $1,00(0,99-1,01)$ \\
\hline Tempo de serviço na SMS (aumento de 1 ano) & $1,00(0,99-1,01)$ & $1,00(0,99-1,01)$ & $1,00(0,99-1,01)$ & $1,00(0,98-1,02)$ & $1,00(0,99-1,01)$ & $1,00(0,99-1,02)$ \\
\hline $\begin{array}{l}\text { Número de consultas semanais (aumento de } 10 \\
\text { consultas) * }\end{array}$ & & & $1,08(1,06-1,11)$ & $1,04(1,00-1,07)$ & & \\
\hline
\end{tabular}

† Razão de prevalências (RP) estimada usando modelo de regressão de Poisson robusta. * Apenas para o modelo estratificado por profissão: médicos. ** Apenas para 0 modelo geral: médicos + enfermeiros. ${ }^{\star * \star}$ Apenas para o modelo estratificado por profissão: enfermeiros. 
Nos modelos multivariáveis estratificados, entre os médicos, a presença de alto escore geral da APS estava associada positivamente com unidade com ESF ( $\mathrm{RP}=1,42$; IC95\%: 1,13-1,79) e número de consultas semanais (para um aumento de 10 consultas semanais, $\mathrm{RP}=1,04$; IC95\%: 1,00-1,07). O tempo de formado, possuir formação em medicina de família, carga horária semanal e tempo de serviço na SMS não apresentaram associaçáo significativa. Para os enfermeiros, possuir formação em enfermagem comunitária ( $\mathrm{RP}=1,16$; IC95\%: 1,05-1,29) estava associado positivamente com a presença de alto escore geral da APS, mesmo ajustando para o tempo de formado, carga de trabalho semanal, tempo de serviço na SMS e tipo de serviço.

\section{Discussão}

Os resultados deste estudo evidenciam que, em Curitiba, na avaliação dos profissionais médicos e enfermeiros, as unidades com ESF possuem maior presença e extensão dos atributos da Atenção Primária. As unidades ESF de Curitiba parecem apresentar maior proporção de profissionais com formação específica em medicina de família e comunidade do que o relatado em estudos realizados com profissionais do estado do Rio Grande do Sul ${ }^{17}$ e também da cidade de Porto Alegre ${ }^{18}$, e sugerem apontar para o maior interesse na formação e em titulação específica na área pelos profissionais das unidades com ESF de Curitiba.

Além disso, os resultados observados pela distribuição da carga horária em relação às atividades diárias desenvolvidas sugerem que nas unidades que atuam com ESF as açóes educativas, as visitas domiciliares e a discussão de casos são mais frequentes, demonstrando, embora timidamente, uma mudança de prática das equipes da ESF. A permanência dos profissionais das equipes durante oito horas diárias nas unidades com ESF parece contribuir para que sejam desenvolvidas outras atividades além das consultas espontâneas ou programadas.

Os altos índices de satisfação apresentados nos dois modelos (acima de 73,6\% em todos os quesitos) demonstram que os profissionais médicos e enfermeiros da APS em Curitiba possuem bom vínculo com o trabalho, com o coordenador, com a equipe e com a comunidade.

Estudos realizados por Guanais e Macinko ${ }^{19,20}$ têm demonstrado que a expansão da Estratégia Saúde da Família tem grande relação com a diminuição das taxas de mortalidade infantil e dos internamentos por condiçóes sensíveis à atenção ambulatorial como diabetes e problemas respiratórios, devido à melhoria do acesso da população à atenção primária. Porém, no presente estudo, a acessibilidade é o atributo com menor escore médio e, apesar do melhor resultado nas unidades com ESF, não atinge o nível de 6,6 (ponto de corte), sendo assim considerado um baixo escore. Observando vários estudos que utilizaram o PCATool, verificamos que os resultados não são homogêneos. Nos estudos com usuários e profissionais de saúde realizados em Petrópolis ${ }^{21}$ e no município de Sáo Paulo ${ }^{22}$, os resultados obtidos dos profissionais de saúde foram superiores no quesito do acesso quando comparados aos usuários. Ibańez et al..$^{23}$ encontraram resultados semelhantes para o atributo acesso quando pesquisado em usuários e trabalhadores entre unidades ESF e UTRAD, apontando valores medianos para este atributo. Em um estudo realizado em Porto Alegre, Cunha et al. ${ }^{17}$ encontraram alto escore no atributo acessibilidade em ambos os modelos, com uma diferença significativa a favor da ESF. No presente estudo, em consonância com vários estudos nacionais que utilizaram o PCATool, o resultado foi divergente, apontando baixo escore médio. Este atributo também apresentou baixo escore em estudo internacional que utilizou a mesma metodologia e foi realizado por Haggerty et al. ${ }^{24}$, com usuários e profissionais no Quebec. No seu estudo, Haggerty menciona que, em uma pesquisa nacional sobre acesso aos serviços de cuidados de saúde, 18,8\% dos canadenses relataram ter dificuldades para acessar esses serviços para problemas de saúde agudos em 2001, e estes índices aumentaram para $24,2 \%$ em 2003. Outros estudos que utilizam metodologias diversas ${ }^{25}$ reforçam esse resultado, apontando que o primeiro contato dos usuários com o sistema ainda apresenta barreiras a serem superadas.

O atributo acessibilidade é avaliado no PCATool, em alguns itens, considerando a disponibilidade da equipe para o atendimento ao usuário em horários fora do atendimento normal da Unidade de Saúde, como períodos noturnos e finais de semana, principalmente relacionados a eventos agudos. No entanto, isto não corresponde à realidade do modelo de atenção primária aplicado no Brasil, o que nos faz refletir sobre a necessidade de adaptação do instrumento de avaliação ou de uma mudança no modelo de atenção, aumentando a disponibilidade do serviço ao cidadáo, mantendo o vínculo e diminuindo a procura por serviços de pronto-atendimento.

É conveniente ressaltar que a organização dos serviços da APS de Curitiba apresenta diferenças importantes em relação a outros locais onde o PCATool foi aplicado, com estruturas de APS padronizadas com atendimento de segunda a sexta-feira no período diurno. Em Curitiba, o sistema municipal de saúde está estruturado com centros municipais de urgências médicas, que atendem às demandas dos usuários nos horários em que as unidades não estão abertas, mas aspectos de acessibilidade próprios das unidades de APS de Curitiba devem ser revisados a fim de que se garanta esse atributo essencial. 
Em relação à longitudinalidade, o presente estudo revela baixo escore nas unidades tradicionais e alto escore nas unidades com ESF, sugerindo que os profissionais destas equipes percebem maior vinculação dos usuários aos serviços, sendo capazes de reconhecer melhor sua população eletiva. Este resultado é semelhante aos encontrados nos estudos realizados em municípios do Estado de São Paulo por Ibañez et al. ${ }^{23}$. Nos resultados apresentados por Elias et al. ${ }^{22}$, referentes ao município de São Paulo, foi encontrada diferença significativa entre as unidades com ESF e as unidades tradicionais apenas no estrato de maior exclusão.

Por outro lado, os atributos da coordenação do cuidado e da coordenaçáo do sistema de informação obtiveram alto escore nos dois modelos de atenção, o que demonstra a importância do investimento feito pelo município no sistema informatizado, que é um elemento facilitador, seja nos resultados dos exames laboratoriais de análises clínicas disponibilizados on-line no prontuário, ou na operacionalização da marcação de consultas especializadas. O resultado do atributo coordenação do cuidado em Curitiba é semelhante ao encontrado na regiáo Centro-Oeste ${ }^{26}$, mas difere dos estudos em municípios do Estado de São Paulo, em que Ibańez classifica como "insuficientes", semelhantemente aos resultados de Porto Alegre relatados por Oliveira ${ }^{15}$ e Cunha et al. ${ }^{17}$.

No atributo da integralidade/serviços disponíveis, foram observados escores médios elevados nos dois modelos, ainda que com melhor desempenho nas unidades com ESF, em contraposição à integralidade/serviços prestados, em que as unidades tradicionais apresentaram baixo escore e as unidades com ESF, alto escore.

Em ambos os modelos, os atributos orientação comunitária e orientação familiar obtiveram altos escores, diferindo de outros estudos realizados no País, nos quais, apesar do melhor resultado das unidades com ESF, os valores encontrados são caracterizados como de baixo escore $e^{22,23,26}$. O alto desempenho encontrado em Curitiba pode ser atribuído aos princípios incorporados na construção da APS no município, como a territorialização, a vigilância à saúde e a responsabilização sanitária, presentes em ambos os modelos.

$\mathrm{Na}$ avaliação do escore geral, que abrange todos os atributos, as UTRADS obtiveram, em média, escore de 6,7 e as unidades com ESF obtiveram o alto escore de 7,4, apontando maior presença e extensão dos atributos da APS neste último modelo, em Curitiba, na visão dos profissionais. Em Porto Alegre, em um estudo realizado com usuários, a avaliação do escore geral relatada por Oliveira ${ }^{15}$ também aponta melhor resultado nas unidades com ESF, apesar de nos dois modelos os valores encontrados serem inferiores aos apresentados neste estudo, em Curitiba.

Os modelos multivariáveis, considerando conjuntamente médicos e enfermeiros (Modelo Geral) e estratificado com médicos, evidenciam que o aumento da prevalência de Alto Escore Geral da APS está associado com o tipo de unidade (ESF). O maior número de consultas médicas semanais também aparece associado com o aumento da prevalência do Alto Escore Geral da APS no modelo estratificado para médicos.

\section{Conclusão}

Os resultados revelam melhor desempenho dos atributos da APS nas unidades com ESF, o que está relacionado, entre outros fatores, com a melhor qualificação dos seus profissionais na APS. Na análise comparativa entre as unidades tradicionais e as unidades com ESF, os resultados sugerem melhor desempenho das unidades com ESF. No entanto, um aspecto a ser considerado neste estudo está nas características peculiares da APS no município de Curitiba, com uma estruturação de UTRAD que não corresponde à organização encontrada em outros municípios brasileiros, não permitindo que os resultados sejam generalizados.

\section{Referências}

1. Starfield B. Atenção primária: equilíbrio entre necessidades de saúde, serviços e tecnologia. Brasília: UNESCO, Ministério da Saúde; 2002. Disponível em: http://bvsms.saude.gov.br/bvs/publicacoes/atencao_primaria_p1.pdf

2. Gené-Badia J, Ascaso C, Escaramis-Babiano G, Sampietro-Colom L, Catalan-Ramos A, Sans-Corrales M, et al. Personalised care, access, quality and team coordination are the main dimensions of family medicine output. Fam Pract. 2007 Fev; 24 (1): 41-7. PMid:17079249. http://dx.doi. org/10.1093/fampra/cml056

3. Haggerty J, Burge F, Lévesque JF, Gass D, Pineault R, Beaulieu MD, et al. Operational definitions of attributes of primary health care: consensus among Canadian experts. Ann Fam Med. 2007 Jul-Ago; 5(4): 336-44. http://dx.doi.org/10.1370\%2Fafm.682 
4. Brasil. Conselho Nacional de Secretários Municipais de Saúde - CONASEMS. Movimento sanitário brasileiro na década de 70: a participação das universidades e municípios - Memórias. Brasília: Athalaia; 2007. 92 p. Disponível em: http://observasaude.fundap.sp.gov.br/BibliotecaPortal/Acervo/ Controle\%20e\%20Participa\%C3\%A7\%C3\%A30\%20Social/Estudos\%20e\%20artigos\%20correlatos/Lvr_MovSantDec70.pdf

5. Brasil. Ministério da Saúde. Relatório Final da 8th Conferência Nacional de Saúde; VIII Conferência Nacional de Saúde; 1986; Brasília. Brasília: MEC; 1986. Disponível em: http://portal.saude.gov.br/portal/arquivos/pdf/8_CNS_Anais.pdf

6. Brasil. Constituição 1988. Constituição da República Federativa do Brasil. Brasília: Senado Federal; 1988. [acesso em 18/12/2008]. Disponível em: http://www.senado.gov.br/sf/legislacao/const/

7. Brasil. Ministério da Saúde. Saúde, Brasil. 147. ed. Brasília: Ministério da Saúde; 2008. Disponível em http://bvsms.saude.gov.br/bvs/periodicos/ saude_brasil_agosto_2008.pdf

8. Brasil. Ministério da Saúde. Atenção básica e a saúde da família: diretriz conceitual. Brasília: Ministério da Saúde; 2004. Disponível em: http://dab. saude.gov.br/atencaobasica.php\#diretriz

9. Ducci L. O sistema de saúde em Curitiba. In: Ducci L, Pedotti MA, Simão MG, Moysés SJ. Curitiba: a saúde de braços abertos. Rio de Janeiro: CEBES, Secretaria Municipal da Saúde de Curitiba; 2001. p. 15-19.

10. Silveira $A D F^{\circ}$, Oliveira $C A$, Ribeiro EM, Lopes MGD. Programa Saúde da Família em Curitiba: estratégia de implementação da vigilância à saúde. In: Ducci L, Pedotti MA, Simão MG, Moysés SJ. Curitiba: a saúde de braços abertos. Rio de Janeiro: CEBES, Secretaria Municipal da Saúde de Curitiba; 2001. p. 239-51.

11. Szwarcwald CL, Luhm KR, Oliveira VLA. "Vigiando" e promovendo a saúde no território municipal. In: Ducci L, Pedotti MA, Simão MG, Moysés SJ. Curitiba: a saúde de braços abertos. Rio de Janeiro: CEBES, Secretaria Municipal da Saúde de Curitiba; 2001. p. 121-3.

12. Shi L, Starfield B, Xu J. Validating the adult primary care assessment tool. J Fam Pract. 2001; 50(2): 161-75.

13. Harzheim E, Duncan BB, Stein AT, Cunha CRH, Goncalves MR, Trindade TG, et al. Quality and effectiveness of different approaches to primary care delivery in Brazil. BMC Health Serv Res. 2006A; 6(156). PMid:17147819 PMCid:PMC1790713. http://dx.doi.org/10.1186/1472-6963-6-156

14. Harzheim E, Starfield B, Rajmil L, Alvarez-Dardet C, Stein AT. Consistência interna e confiabilidade da versão em português do Instrumento de Avaliação da Atenção Primária (PCATool-Brasil) para serviços de saúde infantil. Cad Saude Publica. 2006 Ago; 22(8): 1649-59. http://dx.doi. org/10.1590/S0102-311X2006000800013

15. Oliveira MMC. Presença e extensão dos atributos da atenção primária à saúde entre os serviços de atenção primária em Porto Alegre: uma análise agregada. [Dissertação]. Porto Alegre: Universidade Federal do Rio Grande do Sul; 2007. Disponível em: http://www.lume.ufrgs.br/handle/10183/12649

16. Spiegelman D, Hertzmark E. Easy SAS calculations for risk or prevalence rations and differences. Am J Epidemiol. 2005 Ago; 162(3): 199-200. http:// dx.doi.org/10.1093/aje/kwi188

17. Cunha $\mathrm{CRH}$, Harzheim E, Duncan BB. Percepção da qualidade da atenção à saúde infantil pelos médicos e enfermeiros: comparação entre o Programa Saúde da Família e o modelo tradicional. [Dissertação]. Porto Alegre: Universidade Federal do Rio Grande do Sul; 2006. Disponível em: http://www.lume.ufrgs.br/handle/10183/13684

18. Silva VS, Harzheim E, Castro ED Fo, Agostinho MR, Esperandio E, Soares GV, et al. Perfil dos profissionais que atuam na Estratégia Saúde da Família participantes do Projeto Telessaúde-RS. Rev HCPA. 2008; 28(Suppl):147. Disponível em: http://www.hcpa.ufrgs.br/content/view/1614/1123/

19. Guanais F, Macinko J. Primary care and avoidable hospitalizations: evidence from Brazil. J Ambul Care Manage. 2009; 32(2): 78-9. PMid:19305223. http://dx.doi.org/10.1097/JAC.0b013e31819942e51

20. Macinko J, Guanais F, Souza MFM. Evaluation of the impact of the Family Health Program on infant mortality in Brazil, 1990-2002. J Epidemiol Community Health. 2006; 60: 13-9. PMid:16361449 PMCid:PMC2465542. http://dx.doi.org/10.1136/jech.2005.038323

21. Macinko J, Almeida C, Sá PK. A rapid assessment methodology for the evaluation of primary care organization and performance in Brazil. Health Policy Plan. 2007; 22(3): 167-77. http://dx.doi.org/10.1093/heapol/czm008

22. Elias PE, Ferreira CW, Alves MCG, Cohr A, Kishima V, Escrivão A Jr, et al. Atenção básica em saúde: comparação entre PSF e UBS por estrato de exclusão social no município de São Paulo. Cad Saúde Pública. 2006 Jul-Set;11(3): 633-41. http://dx.doi.org/10.1590/S1413-81232006000300012

23. Ibañez N, Rocha JSY, Castro PC, Ribeiro MCSA, Forster AC, Novaes MHD, et al. Avaliação do desempenho da atenção básica no estado de São Paulo. Ciênc Saúde Coletiva. 2006 Jul-Set; 11(3): 683-703. http://dx.doi.org/10.1590/S1413-81232006000300016

24. Haggerty JL, Pineault R, Beaulieu MD, Brunnelle Y, Gauthier J, Goulet F, et al. Practice features associated with patient-reported accessibility, continuity and coordination of primary health care. Ann Fam Med. 2008; 6(2): 116-23. PMid:18332403 PMCid:PMC2267415. http://dx.doi.org/10.1370/afm.802

25. Souza ECF, Vilar RLA, Rocha NSPD, Uchoa AC, Rocha PM. Acesso e acolhimento na atenção básica: uma análise da percepção dos usuários e profissionais de saúde. Cad Saúde Pública. 2008; 24(Suppl 1):s100-s10. PMid:18660895. http://dx.doi.org/10.1590/S0102-311X2008001300015

26. Stralen CJ, Belisário SA, Stralen TBS, Lima AMD, Massote AW, Oliveira CL. Percepção dos usuários e profissionais de saúde sobre atenção básica: comparação entre unidades com e sem saúde da família na região centro-oeste do Brasil. Cad Saúde Pública. 2008; 24(Suppl 1): s148-s58. PMid:18660899. http://dx.doi.org/10.1590/S0102-311X2008001300019 